\title{
Evolution du dépôt de clapage du Kannick
}

\author{
Philippe Sergent $(*)$, Bainian Zhang $(* *)$ \\ Centre d'Etudes Techniques Maritimes Et Fluviales (*) \\ 2, boulevard Gambetta BP 6003960321 Compiègne cedex \\ Tel :0344926030 Email : philippe.sergent@equipement.gouv.fr \\ Université de Technologie de Compiègne (**) \\ 60, avenue de Landshut 60200 Compiègne \\ Tel : 0344234155 Email : Bainian.Zhang@utc.fr
}

\begin{abstract}
Résumé : Cet article présente le calcul de l'évolution à long terme d'un dépôt de clapage, soumis aux marées, à l'aide d'un modèle numérique aux éléments finis prenant en compte l'évolution des fonds ainsi que le transport du panache en suspension né de l'érosion du dépôt aux pics de flot et de jusant. Les calculs sont comparés à des essais par traceurs radioactifs réalisés en 1989 par le CEA sur le site de clapage du Kannick. Une étude de sensibilité est menée sur les paramètres de la loi érosion-dépôt afin d'ajuster le coefficient $\alpha$ et le coefficient de rugosité de Chezy.
\end{abstract}

Abstract: This paper presents a numerical model, using finite elements, of the long term evolution of a deposit, forced by tides, after dumping of a mixture mudsand in sea. This model takes into account the evolution of the sea bed and the transport in suspension of sediments eroded from the deposit by flux and ebb. The numerical results are compared with experimental data with radioactive tracers coming from tests of CEA in 1989 on the dumping site of Kannick. A sensivity test is performed on parameters of the erosion-deposit law in order to adjust the coefficient $\alpha$ and the Chezy's resistance coefficient.

\section{$\underline{1 . \text { Introduction }}$}

Afin de maintenir les profondeurs navigables dans son chenal d'accès, le port de Rouen doit draguer les fonds en permanence. Les matériaux dragués, composés à $70 \%$ de sable, sont ensuite rejetés en mer par clapage sur des sites de dépôt bien définis. Dans le but de mieux comprendre les mouvements sédimentaires et l'évolution des dépôts ainsi que d'optimiser la gestion des dragages, le port de Rouen a confié une étude de suivi d'un dépôt par traceurs radioactifs au CEA (Commissariat à l'Energie Atomique) en 1989. Ces essais ont été réalisés par période de beau temps, c'est-à-dire avec une influence négligeable des vents et de 
la houle sur le transport des sédiments par rapport à la marée. Les essais par traceurs fournissent des informations très riches sur le processus de mouvements sédimentaires. Dans cette étude, nous cherchons à exploiter ces mesures pour valider des outils de modélisation numérique de l'évolution des dépôts de clapage. Nous présentons d'abord les essais CEA, puis décrivons le principe de modélisation pour enfin comparer les résultats des calculs avec les essais.

\section{$\underline{\text { 2. Mesures par traceurs radioactifs }}$}

Le port Autonome de Rouen réalise des dragages d'entretien pour maintenir la navigabilité du chenal dans l'estuaire de la Seine. Les matériaux dragués sont ensuite clapés sur le site du Kannick. Le port Autonome a confié une étude au CEA (Hoslin et al., 1989) afin d'évaluer le devenir, sur une durée de plusieurs mois de la fraction sableuse des matériaux immergés sur le site du dépôt intermédiaire proche de Honfleur et le site du Kannick. Dans cette étude, nous nous intéressons uniquement au site du Kannick. Une faible masse de traceur radioactif, l'Iridium 192, de période $T 1 / 2$ de 74 jours, a été déposée sur les fonds en deux points distants d'environ deux kilomètres sur une ligne nord-sud, de part et d'autre de la zone de dépôt des produits de dragage. La granulométrie des traceurs est comprise entre 100 et $300 \mu \mathrm{m}$.

Les mesures ont ensuite été réalisées à partir de vedettes du Port Autonome de Rouen équipées de sondes de détection de radioactivité. Elles ont eu lieu sur une période de quatre mois, de mai à septembre 1989 et ont produit notamment des courbes d'isoconcentration en activité, exprimées en chocs par seconde, des diagrammes de transport ainsi que des courbes d'activités cumulées le long de l'axe principal de transit. Les immersions ont eu lieu le 25 mai 1989 à 14 h50 avec un coefficient de marée de 65 au point 1, appelé aussi Kannick Nord, par 11,2 m de fond et le même jour à $16 \mathrm{~h} 00$ au point 2, appelé aussi Kannick Sud, par 8,1 m de fond.

Six détections ont été effectuées aux dates suivantes : 26 mai, 29 mai, 13 juin, 26 juillet, 22 août et 27 septembre. L'axe principal de transit est orienté Sud-Est pour les deux points. Les principaux résultats sont pour le point 1, Kannick Nord, un déplacement général par charriage des sédiments vers l'est, ils pénètrent dans l'estuaire. Avec les coefficients de marées importants du mois d'août supérieurs à 100 , la mise en suspension se généralise. Les résultats au point 2, Kannick Sud, situé seulement à deux kilomètres au sud du point 1 , sont sensiblement différents. Après un mouvement général vers l'est, le mouvement s'oriente vers l'ouest à partir de la période des vives eaux d'août.

A noter enfin que des essais complémentaires ont montré que $75 \%$ de l'activité était retrouvée le jour qui suit le clapage, soit $90 \%$ des sables. On peut donc bien considérer que la quasi-totalité de la fraction sableuse est présente sur le fond après le clapage. 


\section{$\underline{\text { 3. Courantologie }}$}

Les courants bidimensionnels, moyennés sur la verticale, et la hauteur d'eau sont calculés à l'aide du logiciel REFLUX qui résout les équations de Saint-Venant par la méthode des éléments finis (Tanguy et al., 1994).

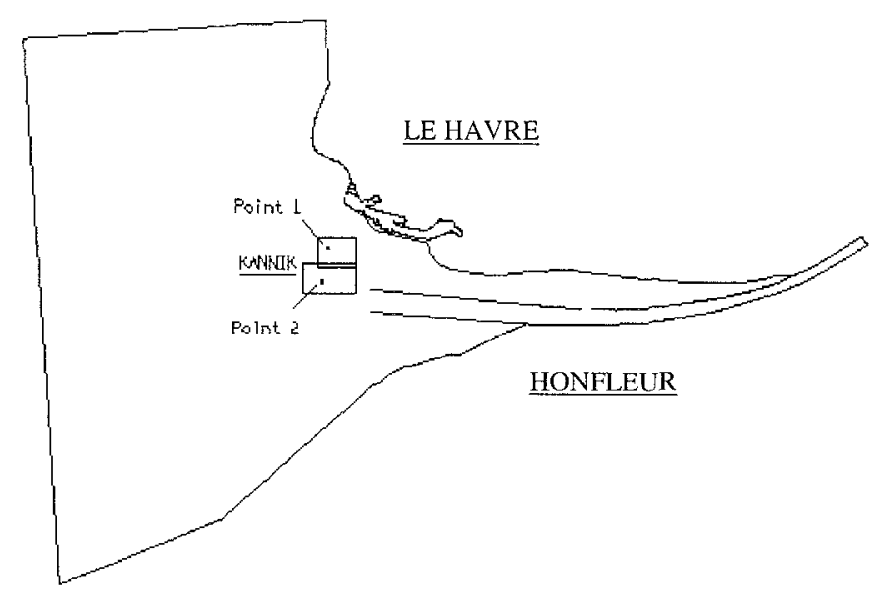

Figure 1: Domaine de calcul - Calculation area.

Le domaine de calcul est présenté sur la figure 1. La condition aux limites côté Manche est du type hauteur d'eau imposée $h(t)$ variant en fonction du temps. Cette loi est celle prise en compte par la SOGREAH lors de son étude de juillet 1989 pour des marées de vives eaux (coefficient 95) et des marées de mortes eaux (coefficient 45). Les conditions aux limites côté Seine, données sur une section située en aval immédiat du pont de Tancarville, sont aussi du type hauteur imposée $\mathrm{h}(\mathrm{t})$. Le modèle a été calé avec un coefficient de Chezy égal à $43 \mathrm{~m}^{1 / 2} \mathrm{~s}^{-1}$.
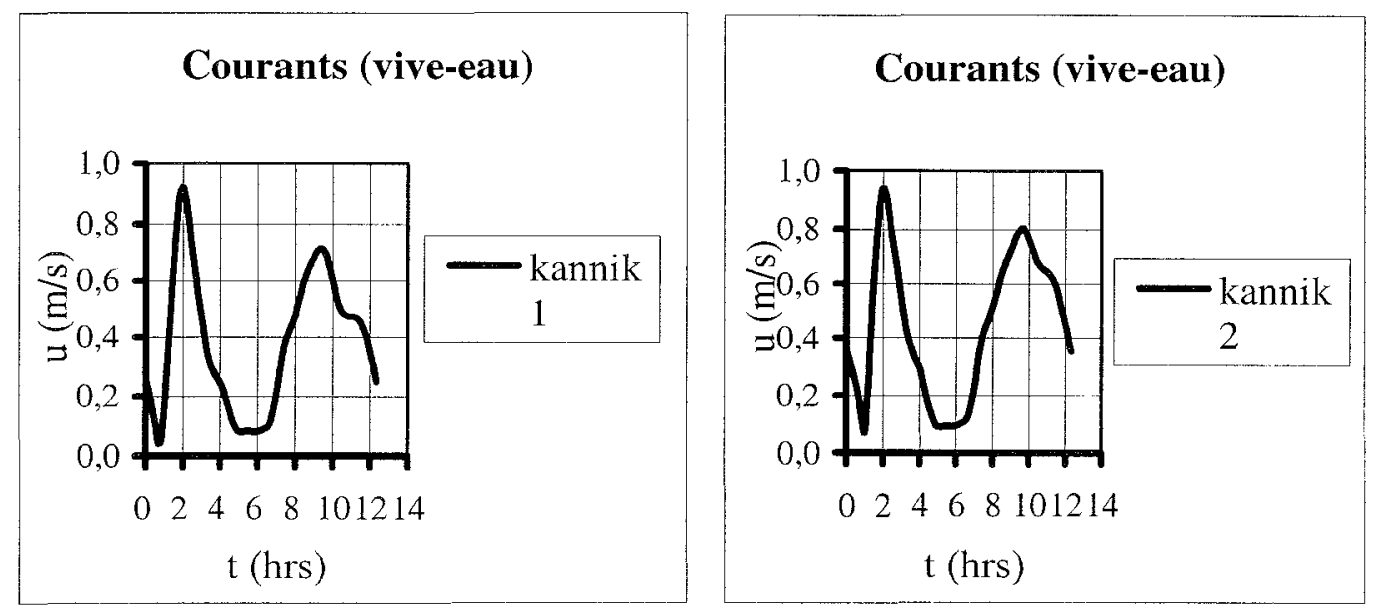

Figure 2: Hydrodynamique sur les lieux de dépôts - Hydrodynamics on the deposit points. 
Deux zones, appelées zone 1 et zone 2, sont extraites du domaine de calcul. Elles contiennent respectivement le point 1, Kannick Nord et le point 2 Kannick Sud. Ces zones mesurent respectivement $2 \mathrm{~km} \times 2 \mathrm{~km}$ et $2,8 \mathrm{~km}$ x $1,6 \mathrm{~km}$. A l'intérieur de chacune, le courant et la vitesse sont quasi homogènes à un instant donné.

La direction des courants est orientée Est-Ouest en jusant et Ouest-Est en flot avec des changements de direction très rapides. Sur la figure 2 sont présentées les vitesses de courant pour des marées de vive-eau (coefficient 95). La durée du jusant est supérieure à celle du flot. La différence de durée entre le jusant et le flot est plus nette pour le point 2 (Kannick Sud) que le point 1 (Kannick Nord). En revanche les vitesses des courants en flot sont supérieures à celles du jusant. Le pic de flot est de $0,9 \mathrm{~m} / \mathrm{s}$ pour les deux points. Le pic de jusant est plus fort au Kannick Sud $(0,8 \mathrm{~m} / \mathrm{s})$ qu' au Kannick Nord $(0,7 \mathrm{~m} / \mathrm{s})$. Le marnage est de huit mètres en vive eau et de quatre mètres en morte-eau. Le point 1 est environ trois mètres plus profond que le point 2 . L'hydrodynamique est surtout marquée, durant la période d'essais (25 mai 1989- 27 septembre 1989), par deux périodes de vives eaux à forts coefficients de marée en Août et Septembre. Les courants et les hauteurs sont calculés pour tous les coefficients de marée à partir des résultats avec coefficients 45 et 95 par interpolation ou extrapolation comme indiqué par le SHOM.

\section{Modélisation de l'évolution du dépôt}

Le dépôt initial est modélisé par un volume de matériaux sableux de forme conique posé sur le fond. Ces sédiments sont ensuite transportés par charriage ou suspension sous l'effet des courants de marée.

\subsection{Equation d'évolution des fonds}

Le débit solide total $\overrightarrow{q_{s}}$ est la somme des débits de charriage et de suspension :

$$
\overrightarrow{q_{s}}=\overrightarrow{q_{s c}}+\overrightarrow{q_{s s}}
$$

L'équation d'évolution des fonds traduit la conservation de la matière. Elle s'exprime de deux façons différentes en fonction du choix de modélisation du transport en suspension. Si le transport en suspension est pris en compte par l'intermédiaire d'une loi de transport donnant le débit solide $\overrightarrow{q_{s s}}$, l'équation d'évolution s'écrit:

$$
\text { (1-p) } \frac{\partial z_{f}}{\partial \mathrm{t}}+\operatorname{div}\left(\overrightarrow{q s c}+\overrightarrow{q_{s s}}\right)=0
$$

Si le transport en suspension est modélisé à travers une équation de convectiondiffusion, l'équation d'évolution prend la forme suivante:

$$
\text { (1-p) } \frac{\partial z f}{\partial t}+\operatorname{div} \overrightarrow{q_{s c}}=-S
$$


avec

- $p$ la porosité des fonds ( $p$ pris égal à zéro),

- $z_{f}$ la cote des fonds,

- $\overrightarrow{q_{s}}$ le transport solide exprimé en $\mathrm{m}^{2} \mathrm{~s}^{-1}$,

- S le terme source représentant le bilan des processus d'érosion et de dépôt des sédiments en suspension.

Nous retenons, dans cette étude, seulement deux lois de transport : la formule de Van Rijn (1984) et celle de Bijker (1968). Ces lois donnent le transport solide en fonction des grandeurs hydrauliques et des caractéristiques des sédiments.

\subsection{Fonds non-érodables}

Ce n'est pas seulement les sédiments immergés en mer mais l'ensemble des sédiments présents sur le fond qui est mis en mouvement sous l'effet des marées et de l'agitation de houle lorsque celle-ci est significative. Les mesures par traceurs radioactifs permettent cependant d'isoler les particules marquées du dépôt du reste des sédiments. Afin de reproduire le résultat des mesures, la modélisation doit elle aussi être capable d'isoler ces sédiments du dépôt. Pour cela on utilise un artifice de modélisation en considérant que les sédiments immergés sont déposés sur un fond non-érodable. On peut ainsi mieux repérer l'évolution du dépôt qui n'est pas masquée par les mouvements d'ensemble des fonds.

Le dépôt initial est modélisé par un cône de rayon 50 mètres et de hauteur 1,5 mètre, soit un volume de matériaux immergés proche de $4000 \mathrm{~m}^{3}$.

Si l'on note $z_{\mathrm{f} 0}$ la cote de référence des fonds avant dépôt, le traitement des fonds non-érodables s'effectue de la façon suivante :

(a) $\operatorname{Si}\left(z_{f}-z_{f 0}\right)<\varepsilon$ et $\operatorname{div} \overrightarrow{q_{s}}>0$ alors $\overrightarrow{q_{s}}=0$

(b) $\quad \operatorname{Si}\left(z_{f}-z_{f 0}\right)<\varepsilon$ et $\left(\operatorname{div} \overrightarrow{q_{s c}}+\mathrm{S}\right)>0$ alors $\overrightarrow{q_{s c}}=0$ et $\mathrm{S}=0$

L'épaisseur $\varepsilon$ est prise égale à $0,5 \mathrm{~mm}$ dans les calculs. Il faut noter que la formulation (a), couramment utilisée, n'est pas totalement satisfaisante car elle implique notamment la conservation de la matière sur le fond autour de la zone de clapage. Comme le volume de matière est conservée, le transport sédimentaire induit un simple étalement du dépôt. Cette conservation de la matière est acceptable pour le transport par charriage mais ne l'est pas pour le transport par suspension. Nous verrons par la suite l'erreur qui peut provenir de cette formulation qui sous-estime le débit de transport solide. Nous privilègierons donc la formulation (b) qui, non seulement prend mieux en compte le transport par suspension mais permet aussi un meilleur traitement des fonds non-érodables. 


\subsection{Equation de transport des sédiments en suspension en 2DH}

L'équation générale de transport de sédiments en suspension en 2D horizontal est exprimée par:

$$
\frac{\partial c}{\partial t}+u 1 \frac{\partial c}{\partial x_{1}}+u 2 \frac{\partial c}{\partial x_{2}}=\frac{\partial}{\partial x_{1}}\left(D_{1} \frac{\partial c}{\partial x_{1}}\right)+\frac{\partial}{\partial x_{2}}\left(D_{2} \frac{\partial c}{\partial x_{2}}\right)+\frac{1}{h} \mathrm{~S}
$$

avec

- $x_{1}, x_{2}$ les coordonnées dans le plan horizontal,

- $u_{1}, u_{2}$ les composantes de la vitesse moyennée sur la verticale,

- $c$ la concentration moyenne des particules sur la verticale,

- $D_{1}, D_{2}$ les coefficients de diffusion des particules solides en suspension. La diffusion est supposée isotrope homogène avec $D_{1}, D_{2}$ égaux à $1 \mathrm{~m}^{2} \mathrm{~s}^{-1}$.

Le terme source est pris égal à $\mathrm{S}=\alpha w_{s}\left(c_{s}-c\right)$ avec $w_{s}$ est la vitesse de chute. Le coefficient $\alpha$ est souvent pris égal à 1, valeur testée par Van Rijn (1985) ou utilisé comme constante homogène de calage (Naceur, 2000). Nous avons, en ce qui nous concerne, choisi d'utiliser soit un coefficient $\alpha$ égal à 1 , soit un coefficient $\alpha$ donné par la formule de Lin et al. (1983) :

- $\alpha=\frac{1}{3}\left(\frac{h}{2 d_{50}}\right)^{\psi_{s} / \psi k u^{*}}$

- avec $\psi$, un coefficient empirique pris égal à $10, \mathrm{k}=0,40$ la constante de Von Karman et $\mathrm{u}^{*}$ la vitesse de cisaillement sur le fond, $u^{*}=\frac{\sqrt{g}}{C_{h}} u$. Dans nos calculs, le coefficient de rugosité de Chezy est soit une constante, prise égale à $60 \mathrm{~m}^{1 / 2} \mathrm{~s}^{-1}$, soit calculé par la formule $C_{h}=18$ $\log \left(\frac{12 h}{3 d_{90}}\right)$

- $c_{s}=0,015 \frac{d_{50}}{k_{s}} \frac{T^{1,5}}{D_{*}^{0,3}}$ la concentration de sable à l'équilibre moyennée sur la verticale, donnée par Van Rijn (1984), $k_{s}$ la rugosité équivalente, T' le nombre de Van Rijn.

\section{Comparaison avec les mesures}

Six types de calculs sont réalisés. La description de chaque calcul est synthétisée dans le tableau suivant: 


\begin{tabular}{|c|c|c|c|c|c|c|}
\hline & A & B & C & D & E & F \\
\hline Méthode & (a) & (a) & (b) & (b) & (b) & (b) \\
\hline Formulation & Van Rijn & Bijker & Van Rijn & Van Rijn & Van Rijn & Van Rijn \\
\hline Chézy & & & constant & variable & constant & variable \\
\hline Coefficient $\alpha$ & & & Lin & Lin & égal à 1 & égal à 1 \\
\hline
\end{tabular}

Tableau 1 : Description des calculs

Les courbes d'isoactivités (mesures) et de bathymétrie (calculs), présentées figure 3 , montrent une différence d'évolution du dépôt entre les sites Nord et Sud. Sur le Kannick Nord, le mouvement est clairement sud-est alors qu'au Kannick Sud, un déplacement dans la direction opposée, nord-ouest, est noté à partir du mois de Juillet.
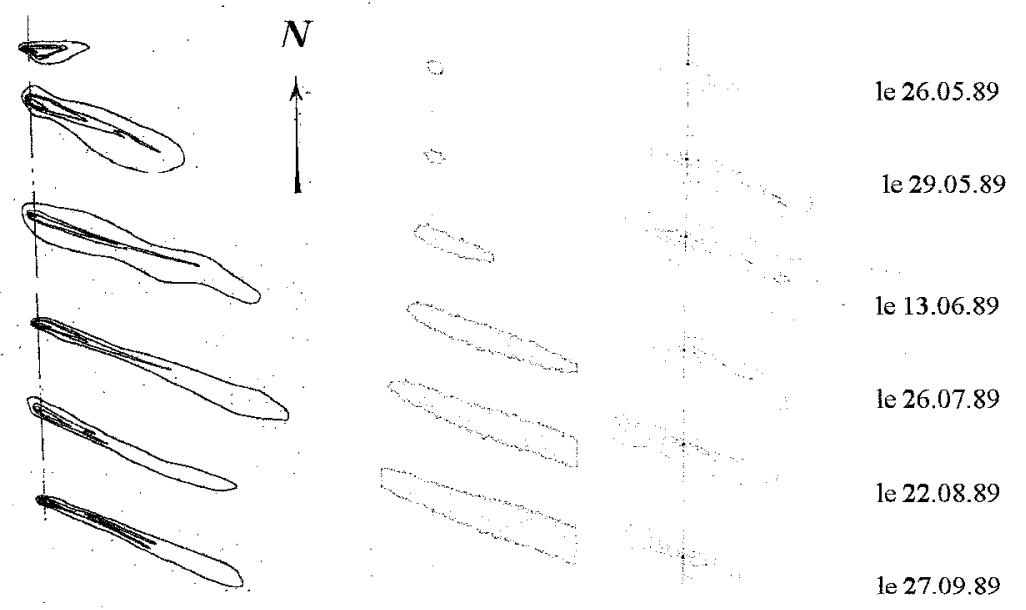

Figure 3 : Courbes d'isoactivités et bathymétrie au Kannick Nord et Kannick Sud - isoactivity curves and bathymetry on North Kannick and South Kannick.

Les courbes d'activités cumulées sur l'axe principal de transit sont présentées sur les figures 4 pour les points 1 et 2 . On constate d'abord sur les mesures au point 1 , une perte de $30 \%$ du volume dans les premières heures des essais. Cette perte de volume, que l'on ne retrouve pas au point 2 , ne peut pas être expliquée par l'hydrodynamique locale. Elle n'est d'ailleurs reproduite dans aucun de nos calculs. On peut imaginer que cette perte s'est produite lors de l'immersion des traceurs et qu'elle n'est pas à mettre sur le compte de l'évolution morphodynamique du dépôt.

D'après les mesures, on constate un transport par charriage qui prédomine pour les coefficients de marée $<100$. A partir du mois d'août, le transport par suspension domine de sorte que l'on ne retrouve plus que $30 \%$ à $50 \%$ des sédiments à la fin des essais, à la fin du mois de septembre. 
Au point 1, le mouvement des matériaux est globalement vers l'Est. Le transport au flot est supérieur au transport au jusant. Au point 2, le bilan entre le flot et le jusant est plus équilibré. On note en particulier un mouvement important vers l'ouest lors des marées à fort coefficient. La différence provient du fait que le pic de jusant est supérieur au point $2\left(0,8 \mathrm{~ms}^{-1}\right.$ contre $\left.0,7 \mathrm{~ms}^{-1}\right)$ et la durée du jusant plus longue.
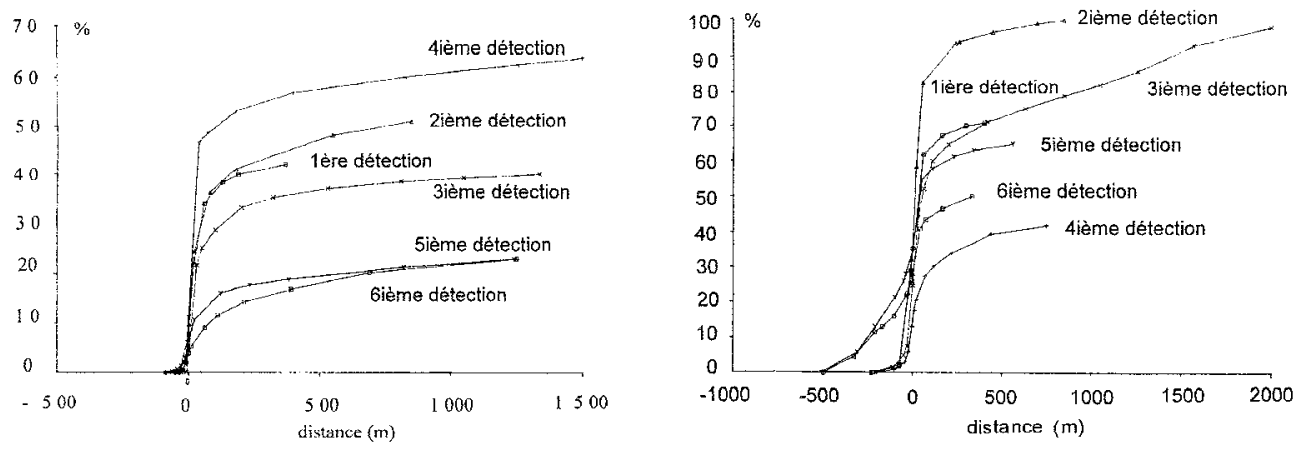

Figure 4 : Courbes d'activités cumulées aux points Kannick Nord et Kannick Sud. Cumulative activity curves on North Kannick and South Kannick.

Les figures 5 et 6 donnent les volumes cumulés sur l'axe principal de transit aux point 1 et 2 respectivement pour les calculs $(\mathrm{A})$ à $(\mathrm{F})$.

Les calculs (A) et (B) sont sensiblement équivalents. On note donc peu de différences entre les formulations de Van Rijn et Bijker. On retrouve bien les différences entre le point 1 et 2 avec le retour des sédiments vers l'ouest au point 2 lors des marées de vives eaux. En revanche, les limites de la formulation des fonds non-érodables sont bien mises en évidence. Quelle que soit l'intensité de la marée, même si le diamètre du dépôt augmente, on retrouve toujours la totalité du volume immergé à l'intérieur du domaine d'étude. Cela est dû au fait que cette formulation conserve le volume des matériaux et induit un simple étalement du dépôt.

Les calculs $(\mathrm{C})$ à $(\mathrm{F})$ permettent de s'affranchir de cette contrainte grâce à la formulation du transport en suspension. Une forte proportion du dépôt est alors érodée et transportée par suspension. L'érosion est trop forte avec le calcul (C). Il reste moins de $10 \%$ aux point 1 et 2 contre des valeurs entre $30 \%$ et $50 \%$ attendus. Le calcul (D) améliore les résultats en réduisant l'érosion. Nous obtenons environ $80 \%$ du volume au point 1 et $40 \%$ du volume au point 2 . Le calcul (E), qui est la formulation la plus simple avec un coefficient $\alpha$ égal à 1 et un coefficient de Chezy constant, semble finalement fournir un bon résultat d'ensemble aux points Nord et Sud. On trouve cependant une érosion légèrement plus forte au Kannick Sud qu'au Kannick Nord alors que les mesures par traceurs donnent une tendance inverse. 

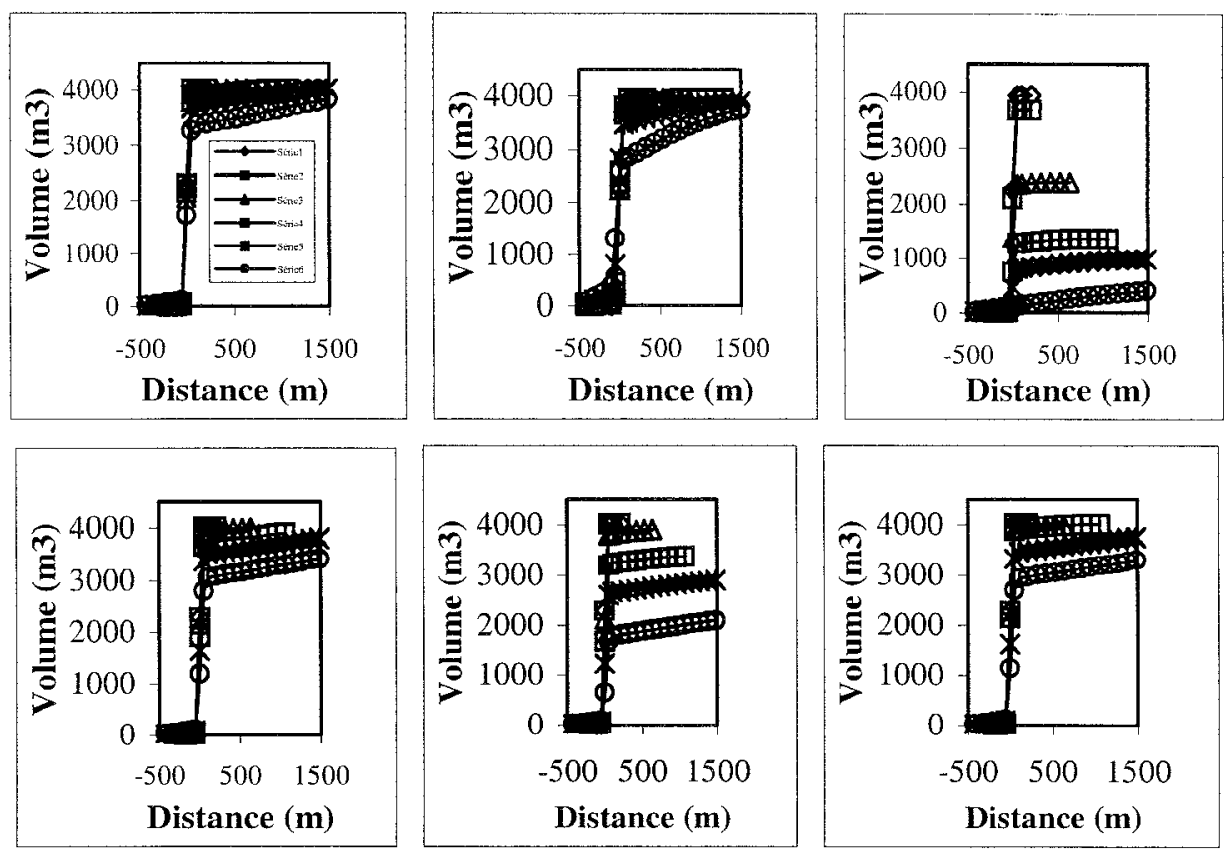

Figure 5: Volumes cumulés au Kannick Nord pour les calculs (A) à (F) Cumulative volumes on North Kannick for the calculations $(A)$ to $(F)$.
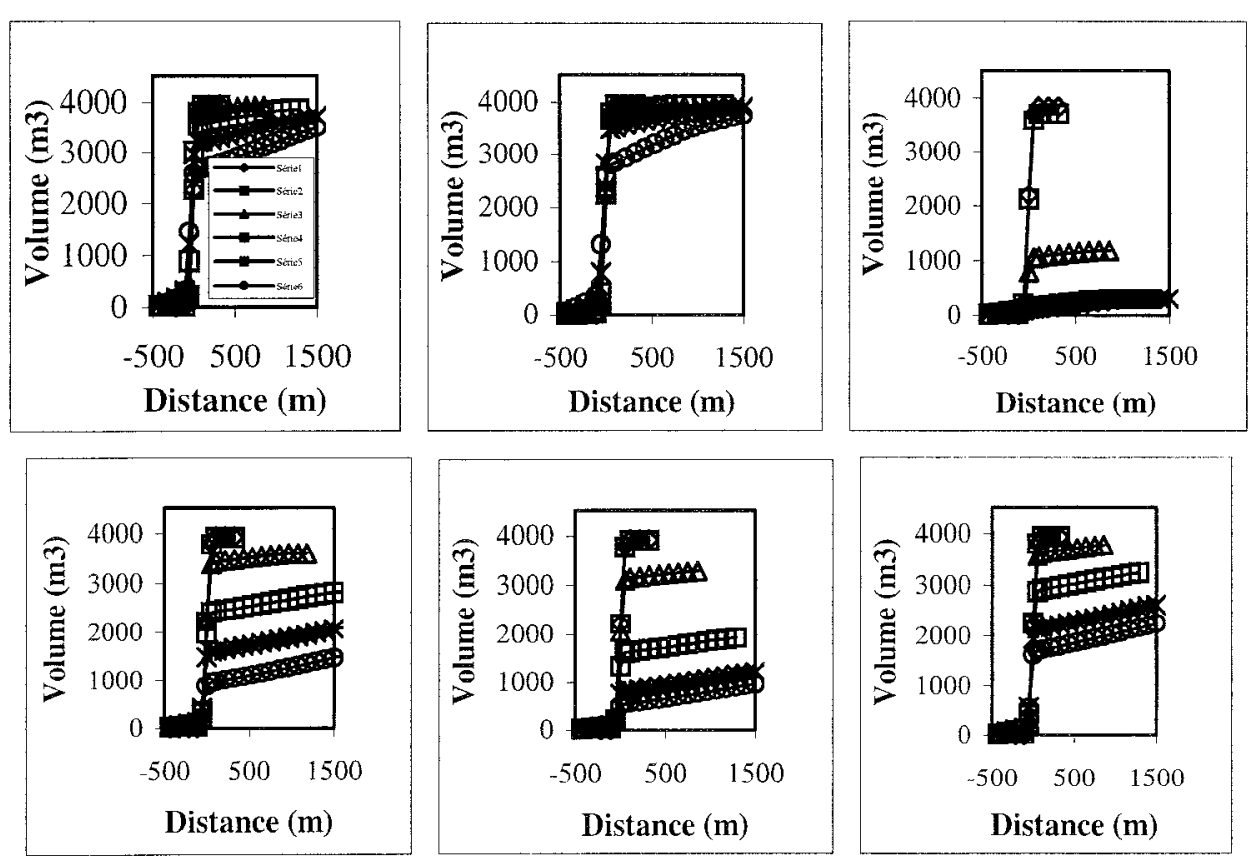

Figure 6: Volumes cumulés au Kannick Sud pour les calculs (A) à (F) Cumulative volumes on South Kannick for the calculations (A) to (F). 


\section{Conclusions}

Cette étude, réalisée dans le cadre du programme LITEAU du MATE en collaboration avec EDF/LNHE et le STTIM, a pour objectif de mettre au point des outils de prédiction du devenir à long terme d'un dépôt de clapage. Les résultats des travaux montrent :

- la sensibilité des transports sédimentaires au lieu de dépôt,

- les limites de la formulation des fonds non- érodables «classique »

- les formulations simples ( $\alpha$ égal à 1 , Chezy constant) fournissent de bons résultats. Un ajustement différencié des coefficients de Chezy devrait pouvoir les améliorer.

Remerciements: Nous remercions le Port Autonome de Rouen de nous avoir donné accès aux résultats des essais par traceurs radioactifs du CEA.

\section{Références}

Hoslin R., Caillot A., SOGREAH, 1990. Estuaire de la Seine - Etude sur la réduction des dragages d'entretien - Expérimentation à l'aide de traceurs radioactifs. Rapport Port Autonome de Rouen

Hoslin R., Brisset P., Massias J., Poggi A., Sannie G., 1989. Etude au moyen de traceurs radioactifs, de l'évolution des dépôts de dragage situés dans l'estuaire de la Seine, zones de dépôt intermédiaire et du vidage aval. Rapport Port Autonome de Rouen.

Hoslin R., Caillot A., Brisset P., Massias J., Poggi A.,Sannie G., 1989. Etude sur la réduction des dragages d'entretien - Expérimentation à l'aide de traceurs radioactifs - Immersion et suivi des traceurs radioactifs à partir d'une drague aspiratrice en marche: clapage et dragage «à l'américaine». Rapport Port Autonome de Rouen

Lin, Pin-Nam, Huan J., Li X., 1983.Unsteady transport of suspended load at small concentrations. Journal of Hydraulic Engineering, ASCE, H109, 1.

Naceur S., 2000. Modélisation tridimensionnelle du transport des sédiments dans les cours d'eau. Thèse de l'Université de Technologie de Compiègne.

SHOM. Courants de marée en Baie de Seine de Cherbourg à Fécamp.

Tanguy J.M, Zhang B., Maine J.J, Marchand B., 1994. Modélisation hydrodynamique de l'estuaire de la Seine - Rapport Port Autonome de Rouen.

Van Rijn L.C., 1984. Sediment transport Part I: Bed load transport, Journal of Hydraulic Engineering, ASCE, 110 (10) 1431-1456.

Van Rijn L.C., 1984. Sediment transport Part II: Suspended load transport, Journal of Hydraulic Engineering, ASCE, 110 (11) 1613-1641.

Van Rijn L.C, 1985. Mathematic modelling of suspended sediment in nonuniform flow, Journal of Hydraulic Engineering, ASCE, 112 (6) 433-455. 